(REVIEW ARTICLE)

\title{
Surgical fire in operating room- A meta synthesis
}

\author{
Raziyeh Ghafouri1,* and MahsaBabaieBashkam² \\ ${ }^{1}$ PhD in Nursing, Assistant professor, Operating Room and Anesthesiology Department, School of Nursing \& Midwifery, \\ Shahid Beheshti University of Medical Science, Tehran, Iran. \\ ${ }^{2} B S$, Operating Room and Anesthesiology Department, Talegani Hospital, Shahid Beheshti University of Medical Science, \\ Tehran, Iran.
}

Publication history: Received on 18August 2020; revised on 29August 2020; accepted on 30August 2020

Article DOI: https://doi.org/10.30574/wjarr.2020.7.2.0310

\begin{abstract}
Operating rooms (ORs) are among the most vulnerable hospital wards to fire and burns. Patient burns are one of the unwanted events that can cause irreparable complications and even death. Therefore, the present study aimed to identify the factors affecting patient burns and propose strategies to prevent patient burns. The present study was a meta-synthesis systematic review. The keywords patient safety, Operating rooms safety, and safe surgery were searched on ProQuest, Ovid, Science Direct, and PubMed. English and Persian articles on fire and patient burns in ORs that were published before 2020 were included. Articles considered suitable by two researchers were analyzed through thematic analysis. Head and neck surgeries are more frequently prone to burns. The most common causes of burns in ORs include lasers, electrocautery, electroshocks, surgical drills, lamps, fiber optic, and warmers. Alcohol-based substances used to disinfect patients' skin are the most important flammable materials in ORs.
\end{abstract}

Keywords: Burn; Fire; Operating room (OR); Safe Surgery

\section{Introduction}

Oxygen-enriched environments are more likely prone to fire [1]. Operating rooms (ORs) ORs are environments rich in oxygen and other flammable gases that can be a source of ignition in exposure to heat [2]. That is why ORs are among the most vulnerable hospital wards to fire and burns. The fire has long been known as one of the unwanted incidents in ORs that not only threatens the medical staff and patients but also can cause irreparable complications and even death [3].

In the US, 650 fires annually occur in ORs [4, 5], most of which cause no complication [3] but 20-30\% of them result in disability or death $[3,6]$. In addition, $1-2 \%$ of fires in OR affect infants every year [3, 7]. Complications of OR fires include severe burns, increased length of stay, increased length of treatment, and increased disability, some of which require further surgeries. This may result in mental traumas in addition to physical injuries in many cases [6].

Fire is a dangerous but preventable phenomenon. Since fire in OR occurs rarely, the medical staff and physicians are not well familiar with its preventive measures [4]. All individuals present in ORs (medical staff, anesthesia staff, surgery team, clients, and patients) are responsible for such incidents [4]. Therefore, the risk of such incidents can be reduced by promoting the medical staff knowledge and establishing effective relationships between them [3, 8]. Gibbs et al. state that training of OR fire prevention to medical staff should be among the top safety priorities [4]. Identification of possible sources and causes of fire in ORs can be the first step in this regard. Therefore, the present study aimed to investigate the factors causing OR fire and propose solutions to prevent it and improve the safety of ORs.

\footnotetext{
* Corresponding author: Raziyeh Ghafouri, Tel:+989127838896; Email: ghafouri@sbmu.ac.ir

PhD in Nursing, Assistant professor, Operating Room and Anesthesiology Department, School of Nursing \& Midwifery, Shahid Beheshti University of Medical Science, Tehran, Iran.
} 


\section{Methods}

In line with the present study objective, all small pieces of existing knowledge about factors affecting fire and patient burns in ORs were collected and evaluated through a comparative approach and meta-synthesis systematic review[911]. Meta-synthesis was conducted based on the strategy proposed by the University of York Center for Reviews and Dissemination Guidance [12].

Since the present study aimed to identify the factors affecting fire and patient burns in ORs, keywords such as patient safety, OR safety, and safe surgery were searched on ProQuest, Ovid, Science Direct, and PubMed. All English and Farsi articles on fire and patient burns in ORs that were published before 2020 were included in the review.

A total of 173 articles were collected. After eliminating the duplicate, 149 articles were selected for the next step. The abstract of articles was reviewed with the inclusion and exclusion criteria and then the eligibility of articles was assessed by the Strengthening the Reporting of Observational studies in Epidemiology" STROBE" and consolidated standards of reporting trials "CONSORT" Review Guidelines. To improve the rigor and trustworthy of the review, the articles were assessed by two separate researchers simultaneously. The articles evaluated suitable by both researchers were analyzed trough thematic analysis. In addition, an audit trial was employed to increase the rigor of the review [9, 10,13]. Figure 1 illustrates the process of articles selection.

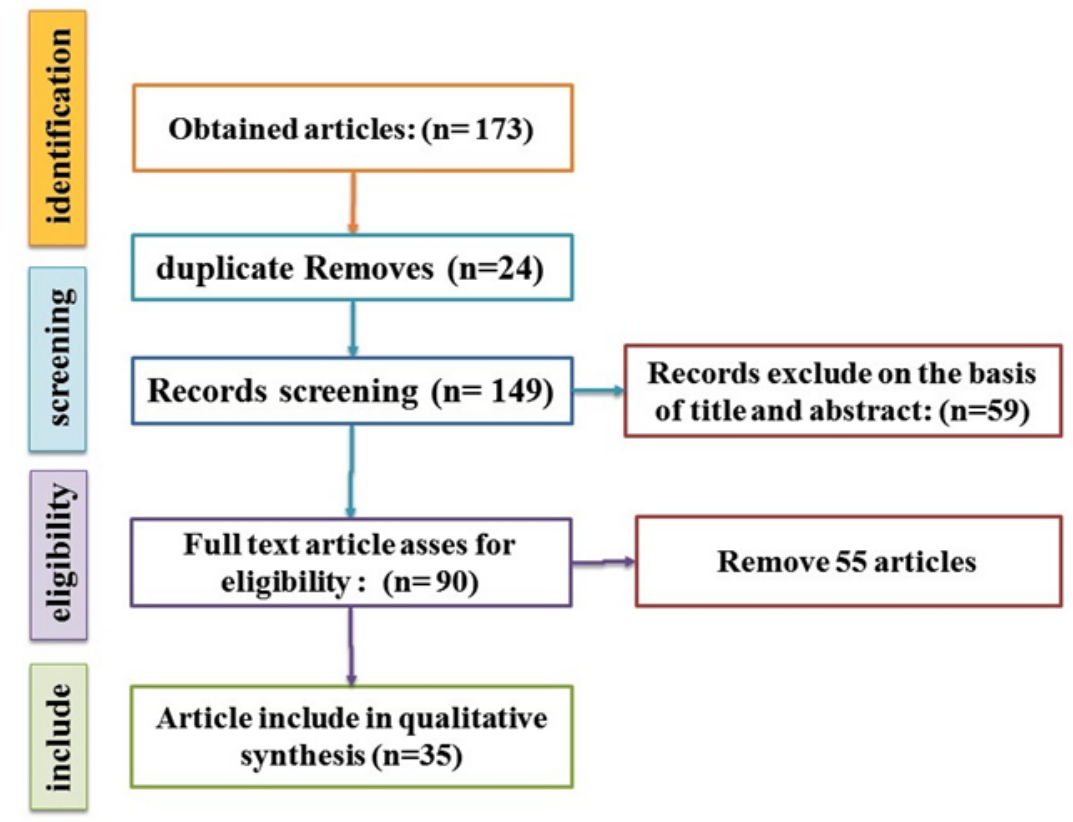

Figure 1 The process of articles selection

\section{Results}

Fire is still considered a potential threat to ORs [3]. Since the triangle of fire consists of fuel, oxygen, and ignition, it can be controlled by removing each side of this triangle [5, 14-16].

The main sources of possible fire in ORs include high levels of oxygen and anesthetic gases $[1,2,5,14,15,17,18]$, flammable materials such as all surgical clothing and drapes, alcohol-based substances used to disinfect patients' skin $[3,5,14,18,19]$, lasers [18, 20-22], electrocautery[20, 21, 23-25], electroshocks [14, 23], surgical drills, lamps [7, 14], fiber optic [20], and warmers [14,15].

To prevent fire and reduce its risk, the best solution is to improve the knowledge and skills of the surgical and anesthesia teams in fire prevention and OR equipment $[3,4,6,8,14,18,25,26]$ and employ appropriate checklists to ensure the safety and soundness of equipment and the accuracy of patient preparation $[4,8,18,27,28]$. Fire safety prevention models in ORs illustrate in figure 2. 


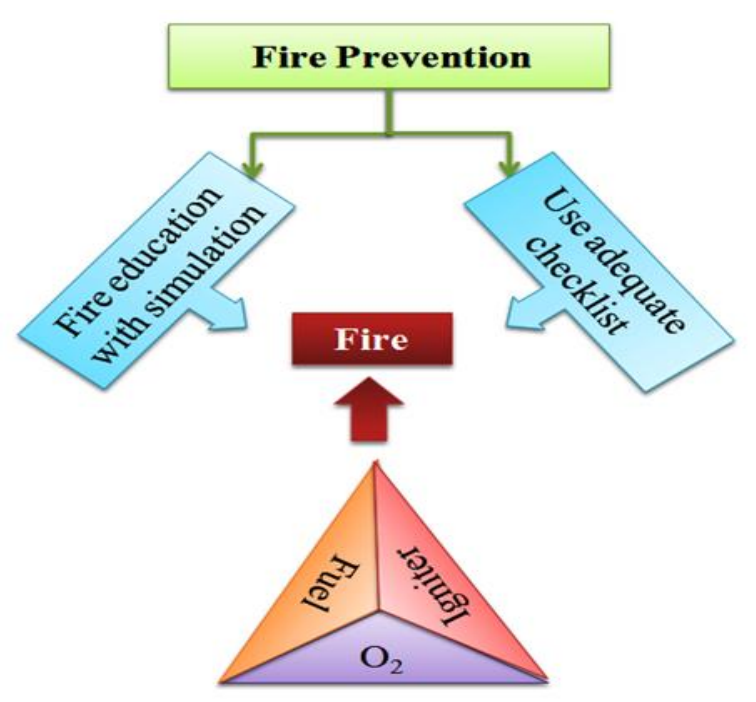

Figure 2 Fire safety prevention models in ORs

\section{Discussion}

The fire has long been known as one of the unwanted incidents in ORs [3]. Root error analysis in fires shows that the main cause of OR fire is the low knowledge of medical staff [17]. Therefore, the risk of OR fire can be reduced by promoting the medical staff knowledge and establishing effective relationships between them [3]. All individuals present in ORs (medical staff, anesthesia staff, surgery team, clients, and patients) are responsible for such incidents [4, 26]. In addition, training of OR fire prevention to medical staff should be among the top safety priorities [4, 8]. Simulation-based learning (SBL) and virtual learning can be helpful in promoting safety knowledge of medical students and staff in simulated real situations without putting patients at risk [6, 25, 29].

The surgical and anesthesia teams should be trained to reduce the concentration of oxygen used to less than $50 \%$ [ $26,30,31$ ], use minimum volume of alcohol-based substances for disinfection (36 mL), especially in head and neck surgeries, and let the disinfectants to get dry in 3 minutes $[5,16]$.

The use of relevant checklists helps to reduce unwanted events and remember the essentials [27, 32] and improves patient safety, safety culture, and efficiency [33-35]. Therefore, it is necessary to use proper checklists to prevent fire in ORs and train the surgical and anesthesia teams in their importance and completion $[8,16,19,28,36]$. As stated by Conley et al., the use of checklists is effective if the medical staff are trained in the importance and completion of them [37].

\subsection{Oxygen}

Oxygen-enriched environments are more likely prone to fire [1]. ORs are environments rich in oxygen and other flammable gases that can be a source of ignition in exposure to heat [2]. Head and neck surgeries are more frequently vulnerable to burns [21]. Most burns caused by high levels of oxygen are observed in surgeries performed above the xiphoid or the third thoracic vertebrae [5]. The highest reports of OR fire belong to tracheostomy [2, 30, 38, 39].

Overbey et al, reported that OR burns can occur in internal organs such as the mouth and $42 \%$ of internal burns result in death [21]. Also they have been reported that 167 cases of OR burns occurred from 1995 to 1998 consisting of 56 cases (32\%) in the throat and mouth, 47 cases (28\%) on the head and neck, and 24 cases (14\%) in internal organs other such as the thorax [7].

Using the minimum oxygen concentration helps to reduce the risk of fire in ORs. It has been shown that the use of 15 liters per minute of $100 \%, 60 \%$, and $50 \%$ oxygen can cause fire in 15-30, 25-60, and 128-184 seconds, respectively. However, 45-50\% oxygen can rarely cause fire [15]. Roy and Smith stated that the use of the 29\% oxygen for laser therapy does not cause fire in the OR [40].

When using high concentrations of oxygen, it is recommended to avoid using alcohol and alcohol-based substances as far as possible and change the position of the operating table to be at the furthest point to the anesthetic bag and machine 
[6]. Oral suctioning also helps reduce oxygen concentration and prevent fire [1]. Although fireproof covers do not completely eliminate the possibility of fire, their use for endotracheal tubes in head and neck and throat surgeries, along with reducing oxygen concentration to $40 \%$, can be effective in prevention of OR fire [40,41]. Although putting a wet sterile gauze at the bottom of the pharynx does not completely prevent fire, it is helpful [40]. The endotracheal tube cuff should be filled with normal saline and kept far away from the laser as much as possible [40, 41]. Increasing the concentration of carbon dioxide may be reduce the risk of fire during tracheostomy [40].

\subsection{Causes of fire}

The most common causes of fire and burns in ORs are lasers [18, 20-22], electrocautery [20, 21, 23-25], electroshocks $[14,23]$, surgical drills, lamps [7, 14], fiber optic [20], and warmers [14, 15]. Although the use of above-mentioned equipment may cause only 1-2 cases of fire in every 1000 surgeries, it should be borne in mind that 50 billion surgeries are performed annually around the world [21].

Monopolar cauterizes were among common surgical equipment during the twentieth century. There were many reports of burns caused by monopolar cauterizes in the period 1920-1970 [20,21]. Bipolar cauterizes reduce the risk of fire and patient burns in ORs [24]. If a monopolar cautery is used in the OR, it is better to use appropriate size plate or a blend plate to reduce the risk of patient burns [14,23]. When high concentrations of oxygen (90-100\%) are used during surgery, it is recommended to turn off the cautery or use a low-voltage, low-energy bipolar one [2, 14, 39].

Technological advances have made it possible to perform surgeries with small incision [31]. However, application of these new practices is not fully risk-free and can harm both patients and the medical staff [20, 25, 31]. One of the rare but dangerous complications of such practices is fire and patient burns during surgeries [3, 20].

The use of carbon dioxide in laparoscopy can reduce the risk of OR fire [3]. According to Odell, the cover of surgical equipment should be checked for possible rupture to prevent unwanted visceral burns in laparoscopic surgeries [20]. It is recommended to use minimum laser energy and a checklist to prevent fire. In addition, precautions should be seriously taken into account to prevent fire during head and neck surgeries [18, 20-22, 40].

\subsection{Flammable materials in the $\mathrm{OR}$}

The most important flammable materials in ORs include surgical clothing and drapes and alcohol-based substances used to disinfect patients' skin $[3,5,14,18,19]$.

Yardley and Donaldson recommend that the substances used in ORs should be labeled to prevent misuse of them [3]. Meltzer et al. emphasize that water-based gels are better to be used in ORs instead of oily gels and ointments [42].

\section{Conclusion}

ORs are among the most vulnerable hospital wards to fire and burns. Using the minimum levels of oxygen, controlling the leakage of anesthesia gases, checking the equipment before surgeries, observing safety tips when using any surgical equipment, using the effective and appropriate amount of disinfectants, drying the patient after using an alcohol-based disinfectant, and preventing the patient's body from getting wet are the most important ways to reduce the risk of patient burns in ORs.

\section{Compliance with ethical standards}

\section{Acknowledgments}

The authors also wish to thank all of Operating rooms staffs of Shahid Beheshti University of Medical Science, Tehran, Iran.

\section{Disclosure of conflict of interest}

The authors declare that they have no competing interests. 


\section{References}

[1] VanCleave AM, Jones JE, McGlothlin JD, Saxen MA, Sanders BJ, Vinson LA. The effect of intraoral suction on oxygenenriched surgical environments: a mechanism for reducing the risk of surgical fires. Anesthesia progress. 2014; 61(4): 155-61.

[2] Lin IH, Hwang CF, Kao YF, Chang KA, Peng JP. Tracheostomal fire during an elective tracheostomy. Chang Gung Med J. 2005; 28(3): 186-90.

[3] Yardley I, Donaldson L. Surgical fires, a clear and present danger. The surgeon. 2010; 8(2): 87-92.

[4] Gibbs VC. Thinking in three's: changing surgical patient safety practices in the complex modern operating room. World Journal of Gastroenterology: WJG. 2012; 18(46): 6712.

[5] Spratt D, Cowles Jr CE, Berguer R, Dennis V, Waters TR, Rodriguez M, et al. Workplace safety equals patient safety. AORN journal. 2012; 96(3): 235.

[6] Mullen L, Byrd D. Using simulation training to improve perioperative patient safety. AORN journal. 2013; 97(4): 419-27.

[7] Bruley ME. Surgical fires: perioperative communication is essential to prevent this rare but devastating complication. BMJ Quality \& Safety. 2004; 13(6): 467-71.

[8] Kaye AD, Kolinsky D, Urman RD. Management of a fire in the operating room. Journal of anesthesia. 2014; 28(2): 279-87.

[9] Speziale HS, Streubert HJ, Carpenter DR. Qualitative research in nursing: Advancing the humanistic imperative: Lippincott Williams \& Wilkins. 2011.

[10] Paterson BL, Thorne SE, Canam C, Jillings C. Meta-study of qualitative health research: A practical guide to metaanalysis and meta-synthesis: Sage. 2001.

[11] Zimmer L. Qualitative meta-synthesis: a question of dialoguing with texts. Journal of advanced nursing. 2006; 53(3): 311-8.

[12] YorkUniversity. Systematic Reviews:CRD's guidance for undertaking reviews in health care. thirded: Centre for Reviews and Dissemination, University of York.York Publishing Services Ltd; 2008 January 2009.

[13] Ghafouri R, Ofoghi S. Trustworth and rigor in qualitative research. International Journal of Advanced Biotechnology of Applied Behavioral Science. 2016; 7: 90-101.

[14] Smith C. Surgical fires-learn not to burn. AORN journal. 2004; 80(1): 23-36.

[15] Roy S, Smith LP. What does it take to start an oropharyngeal fire? Oxygen requirements to start fires in the operating room. International journal of pediatric otorhinolaryngology. 2011; 75(2): 227-30.

[16] Spruce L. Back to basics: preventing surgical fires. AORN journal. 2016; 104(3): 217-24.

[17] Hempel S, Maggard-Gibbons M, Nguyen DK, Dawes AJ, Miake-Lye I, Beroes JM, et al. Wrong-site surgery, retained surgical items, and surgical fires: a systematic review of surgical never events. JAMA surgery. 2015; 150(8): 796805.

[18] Watson DS. New recommendations for prevention of surgical fires. AORN journal. 2010; 91(4): 463-9.

[19] Rocos B, Donaldson L. Alcohol skin preparation causes surgical fires. The Annals of The Royal College of Surgeons of England. 2012; 94(2):87-9.

[20] Odell RC. Surgical complications specific to monopolar electrosurgical energy: engineering changes that have made electrosurgery safer. Journal of minimally invasive gynecology. 2013; 20(3): 288-98.

[21] Overbey DM, Townsend NT, Chapman BC, Bennett DT, Foley LS, Rau AS, et al. Surgical energy-based device injuries and fatalities reported to the food and drug administration. Journal of the American College of Surgeons. 2015; 221(1): 197-205.

[22] Schroeck H, Healy DW, Tait AR. Airway laser procedures in children and the American Society of Anesthesiologists' Practice Advisory: A survey among pediatric anesthesiologists. International journal of pediatric otorhinolaryngology. 2014; 78(12): 2140-4.

[23] Palmer JHM. Surgical diathermy and electrical hazards: causes and prevention. Anaesthesia\& Intensive Care Medicine. 2016; 17(10): 480-5. 
[24] Feldman L, Fuchshuber PR, Jones DB. The SAGES manual on the fundamental use of surgical energy (FUSE): Springer; 2012.

[25] Dorozhkin D, Olasky J, Jones DB, Schwaitzberg SD, Jones SB, Cao CG, et al. OR fire virtual training simulator: design and face validity. Surgical endoscopy. 2017; 31(9): 3527-33.

[26] Lypson ML, Stephens S, Colletti L. Preventing surgical fires: who needs to be educated? The Joint Commission Journal on Quality and Patient Safety. 2005; 31(9): 522-7.

[27] Huang J. The initial steps of operating room emergency checklist. Journal of clinical anesthesia. 2015; 8(27): 6923.

[28] Walker I, Reshamwalla S, Wilson I. Surgical safety checklists: do they improve outcomes? British journal of anaesthesia. 2012; 109(1): 47-54.

[29] Lee MY, Kim SS. A Safety Simulation Program for Operating Room Nurses. Clinical Simulation in Nursing. 2018; 18: 6-13.

[30] Gorphe P, Sarfati B, Janot F, Bourgain J, Motamed C, Blot F, et al. Airway fire during tracheostomy. European annals of otorhinolaryngology, head and neck diseases. 2014; 131(3): 197-9.

[31] Lee J-Y, Park CB, Cho E-J, Kim CJ, Chea JS, Lee BH, et al. Airway fire injury during rigid bronchoscopy in a patient with a silicon stent-A case report. Korean journal of anesthesiology. 2012; 62(2): 184.

[32] Zingiryan A, Paruch JL, Osler TM, Hyman NH. Implementation of the surgical safety checklist at a tertiary academic center: Impact on safety culture and patient outcomes. The American Journal of Surgery. 2017; 214(2): 193-7.

[33] Russ S, Rout S, Caris J, Mansell J, Davies R, Mayer E, et al. Measuring variation in use of the WHO surgical safety checklist in the operating room: a multicenter prospective cross-sectional study. Journal of the American College of Surgeons. 2015; 220(1): 1-11. e4.

[34] Patel J, Ahmed K, Guru KA, Khan F, Marsh H, Khan MS, et al. An overview of the use and implementation of checklists in surgical specialities-a systematic review. International Journal of Surgery. 2014; 12(12): $1317-23$.

[35] Magill ST, Wang DD, Rutledge WC, Lau D, Berger MS, Sankaran S, et al. Changing Operating Room Culture: Implementation of a Postoperative Debrief and Improved Safety Culture. World neurosurgery. 2017; 107:597603.

[36] Castelluccio D. Implementing AORN recommended practices for laser safety. AORN journal. 2012; 95(5): 612-27.

[37] Conley DM, Singer SJ, Edmondson L, Berry WR, Gawande AA. Effective surgical safety checklist implementation. Journal of the American College of Surgeons. 2011; 212(5): 873-9.

[38] Rogers M, Nickalls R, Brackenbury E, Salama F, Beattie M, Perks A. Airway fire during tracheostomy: prevention strategies for surgeons and anaesthetists. Annals of the Royal College of Surgeons of England. 2001; 83(6): 376.

[39] Kim M-S, Lee JH, Lee D-H, Lee YU, Jung TE. Electrocautery-ignited surgical field fire caused by a high oxygen level during tracheostomy. The Korean journal of thoracic and cardiovascular surgery. 2014; 47(5): 491.

[40] Roy S, Smith LP. Surgical fires in laser laryngeal surgery: are we safe enough? Otolaryngology--Head and Neck Surgery. 2015; 152(1): 67-72

[41] Tykocinski M, Thomson P, Hooper R. Airway fire during tracheotomy. ANZ journal of surgery. 2006; 76(3): 1957.

[42] Meltzer HS, Granville R, Aryan HA, Billman G, Bennett R, Levy ML. Gel-based surgical preparation resulting in an operating room fire during a neurosurgical procedure: case report. Journal of Neurosurgery: Pediatrics. 2005; 102(3): 347-9. 